\title{
Custom Made Piggeries in Norway? View of the Meat Producing Industry
}

\author{
By Harald Gjein \\ Norsvin, Box 504, N-2304 Hamar, Norway. \\ Tel.: + 47625101 00, Fax: + 47625101 01, E-mail: harald.gjein@norsvin.no
}

\section{Introduction}

Xenotransplantation is an exciting, yet controversial, subject. In many ways it presents a challenge to Norwegian swine producers. Norwegian swine production wants to market itself to the Norwegian consumer as providing high quality, safe food items. We are putting a lot of effort into securing our high standard of animal health and welfare, including a low prevalence of zoonoses and "clean" food, i.e. meat free of medicinal residues from antibiotics and hormones. Currently, we have a very high standard of animal health in Norway as shown by official free status (OIE) of all serious contagious animal diseases and a very low prevalence of the less serious contagious diseases. As a result of this we have a very restricted use of antibiotics and other animal medicines.

Regarding animal welfare, we have introduced the practice of free roaming sows in grouphousing systems as the major production system, as opposed to individually pen restricted pigs. Furthermore, we have, as the only country in the world so far, prohibited castration of boars, a legislation, which will be effectuated from the year 2009. Until then, only veterinarians may castrate boars, and local anaesthesia is mandatory.

This introduction is to illustrate that the Norwegian swine producers wish to convey that they are concerned about public opinion regarding the quality of pork meat, and that they wish to produce meat that is safe to consume, and is produced from pigs that enjoy a high standard of welfare during their lifetime. From this angle I venture to discuss the issue of xenotransplantation.

\section{Identifying the controversies}

We all know that xenotransplantation raises a lot of questions. One serious challenge is the transmission of infectious agents from pigs to humans. Severe tissue rejection due to incompatibility is another serious matter. Furthermore, the animal welfare issue of rearing and producing pigs as organ source animals is yet another problem that needs to be addressed.

Norsvin, as a representative of the Norwegian swine breeders, clearly see that we are at an advantage to provide conditions for possible production of source pigs, and that we are willing to participate in the development of xenotransplantation should the need arise. Apart from being able to provide optimal general rearing conditions in Norway, such as environments that are virtually free of the serious health hazards caused by the traditional swine pathogens, we still face the problem of endogenous retrovirus associated with the swine genome. Still, a lot of research is needed to solve this problem.

From our point of view a development that introduces the use of pigs as source animals for organs could represent a hazard by changing 
the consumers' attitudes towards pork and their concept of safe food. The risk, however small, that is embedded in the possible transmission of viruses from pigs to humans through xenotransplantation, could lead to the consumers thinking that there is a danger also in eating meat from pigs. Another risk, although maybe not as prominent, is that some people will react negatively due to the pigs being too closely related to themselves, for instance if a family member, or someone they know, has a heart transplant from a pig. Or that they feel that they are more related to pigs than many other animal species, such as illustrated by the massive attention given to the famous killer whale Keiko. Some species of animals seem more naturally disposed to stimulating the human animal bond and raise emphaty. Is it acceptable to eat dog meat, dogs being "man's best friend"?

Then one has the intuitive aversion towards having to eat something from an animal species that is used as an organ source in human medical operations. Also fear or apprehension may arise among some people against eating meat from an animal that they think has been genetically manipulated, in as much a parallel to the reaction observed from the discussion on genetically modified organisms (GMO) in plant production. Some people may have difficulties in distinguishing between some genetically modified pigs made specifically for organ donation and ordinary pigs reared for meat consumption. How do we know that meat from these animals does not hit the supermarket? And then there is the animal welfare aspect related to transgenic pigs and justified concern with respect to the rearing of the animals under conditions that really secure animal welfare. We have to respect that the animals themselves have a value beyond their product.

Last, but not least, the emergence of new swine pathogens, possibly arising from human pathogens or swine pathogens modified through passages in humans, that may lead to new diseases or an epidemic in pigs is not fully elucidated. This area is still speculative, but should be considered.

\section{Conclusion}

Norwegian swine breeders are positive to the use of pigs for the purpose of xenotransplantation, but at the same time they are aware, that the use of pig organs for xenotransplantation may negatively influence the attitudes of the consumers towards pork. 